\title{
Determining the Relative Orientation Between the Chemical Shift Anisotropy and Heteronuclear Dipolar Tensors in Static Solids by SEDOR NMR
}

\author{
Brendan W. Wilson, Arlo A. Parker, and Terry Gullion \\ Department of Chemistry \\ West Virginia University \\ Morgantown, WV 26506
}

Keywords: principal axis system, chemical shift anisotropy, PAS, CSA, SEDOR

\begin{abstract}
$\underline{\text { Abstract }}$
The measurement of the dipolar interaction between two spins provides the distance between nuclei. A better structural picture emerges when the distance is combined with the orientation of the internuclear vector in the principal axis system of the chemical shift anisotropy tensor. The SEDOR experiment is used on a static sample of alanine to show that the orientation of the vector connecting the nitrogen and carboxylate carbon nuclei can be accurately determined in the CSA PAS of the ${ }^{13} \mathrm{C}$ carboxylate spin.
\end{abstract}

(C) 2016. This manuscript version is made available under the Elsevier user license http://www.elsevier.com/open-access/userlicense/1.0/ 


\section{Introduction}

Magic angle spinning nuclear magnetic resonance (MAS NMR) dipolar recoupling provides a way to obtain structural detail in a solid by determination of an internuclear distance through the measured dipolar coupling [1-3]. An enhanced structural picture emerges by finding the orientation of the internuclear vector of the coupled spin pair with respect to the principal axis system (PAS) of the chemical shift anisotropy (CSA) tensor of one of the coupled spins [4-16].

In addition to MAS NMR techniques, there are NMR methods for determining the orientation between the dipolar and chemical shift anisotropy tensors for static solids [1721]. Stoll, Vega, and Vaughn demonstrated in 1976 a ${ }^{1} \mathrm{H}-{ }^{13} \mathrm{C}$ double-resonance experiment, henceforth referred to as SVV, to determine the orientation of the $\mathrm{C}-\mathrm{H}$ bond with respect to the ${ }^{13} \mathrm{C}$ CSA PAS [20]. In SVV, the ${ }^{13} \mathrm{C}$ signal was acquired after application of a standard ${ }^{13} \mathrm{C}$ spin-echo sequence of fixed duration $\tau$. The ${ }^{1} \mathrm{H}$ sequence contained a multi-pulse window of length $t_{1}$ and a decoupling field during the remainder of the $\tau$ period and during ${ }^{13} \mathrm{C}$ signal detection. The multi-pulse window suppressed the proton-proton dipolar interaction while maintaining a finite ${ }^{1} \mathrm{H}-{ }^{13} \mathrm{C}$ heteronuclear dipolar interaction. The result of SVV is that the observed ${ }^{13} \mathrm{C}$ line shape is amplitude modulated by the heteronuclear ${ }^{1} \mathrm{H}_{-}{ }^{13} \mathrm{C}$ dipolar interaction. Simulation of the resulting ${ }^{1} \mathrm{H}$-decoupled ${ }^{13} \mathrm{C}$ line shape provided the desired orientation information. In some aspects, SVV is a version of the Kaplan and Hahn spin-echo double-resonance experiment (SEDOR) [2224] modified to take into account the strong ${ }^{1} \mathrm{H}-{ }^{1} \mathrm{H}$ homonuclear dipolar interaction.

In this short paper, we revisit the SVV experiment. In particular, we show that a ${ }^{13} \mathrm{C}-{ }^{15} \mathrm{~N}$ SEDOR difference experiment can accurately provide the orientation of the ${ }^{13} \mathrm{C}$ ${ }^{15} \mathrm{~N}$ internuclear vector in the PAS of the ${ }^{13} \mathrm{C}$ CSA tensor. Simulations are used to show that the orientation can be determined within $5^{\circ}$. The SEDOR experiment is straightforward, especially for a pair of rare-spin nuclei, and requires only a single dipolar modulated difference spectrum.

\section{Experimental Details and Methods}

Experiments on a static sample were performed on a custom-built spectrometer using a Tecmag Redstone console and a 3.55 tesla magnet (proton frequency of 151.395 
MHz). The NMR probe is a transmission-line design, and it incorporates a Chemagnetics $7.5 \mathrm{~mm}$ pencil rotor spinning assembly with a $14 \mathrm{~mm}$ long, $8.65 \mathrm{~mm}$ inner diameter, 6turn coil made of 14-gauge tinned copper wire. Radio-frequency (rf) field strengths on the proton channel were $115 \mathrm{kHz}$ for ${ }^{1} \mathrm{H}$ decoupling and $50 \mathrm{kHz}$ for ${ }^{1} \mathrm{H}^{13}{ }^{13} \mathrm{C}$ crosspolarization. ${ }^{13} \mathrm{C}$ and ${ }^{15} \mathrm{~N}$ rf field strengths were $50 \mathrm{kHz}$. The ${ }^{1} \mathrm{H},{ }^{13} \mathrm{C}$, and ${ }^{15} \mathrm{~N}$ power amplifiers were under active control. The ${ }^{13} \mathrm{C}$ spectra were obtained with recycle delays of one second and Hartmann-Hahn cross-polarization transfer times of $0.5 \mathrm{~ms}$.

The powder sample was made of a 1.0:8.4 mass ratio of L-alanine $\left(1-{ }^{13} \mathrm{C}, 99 \%\right.$; ${ }^{15} \mathrm{~N}, 98 \%$ ) and natural abundance L-alanine recrystallized from water. The labeled alanine was obtained from Cambridge Isotope Laboratories, Inc., and the natural abundance alanine was obtained from Acros Organics. The ${ }^{13} \mathrm{C}$ spectrum of the carboxylate carbon for this sample is dominated by the chemical shift anisotropy, and the CSA parameters determined from the spectrum of our sample are an asymmetry parameter of 0.77 and an anisotropy of $-2.71 \mathrm{kHz}$. On our spectrometer, the edge-toedge width of the ${ }^{13} \mathrm{C}$ spectrum of the line shape from the carboxylate carbon is approximately $5.1 \mathrm{kHz}$. In this work, a ${ }^{13} \mathrm{C}-{ }^{15} \mathrm{~N}$ dipolar coupling of $195 \mathrm{~Hz}$ was used for simulations; this value is intermediate between a rigid lattice calculation and a REDORdetermined value. Simulations suggest that a $6 \%$ variation in the dipolar coupling has little effect on difference spectrum lineshape. A 1.6 millisecond dipolar evolution period was used for all experiments and simulations. The experimental and simulated spectra are all shown with a spectral width of $10 \mathrm{kHz}$.

NMR simulations were performed with the SpinEvolution software package [25] using the NMR parameters described in the preceding two paragraphs and with a Lorentzian broadening of $140 \mathrm{~Hz}$ and a Gaussian broadening of $300 \mathrm{~Hz}$.

\section{$\underline{\text { Results and Discussion }}$}

Naito et al. [26] determined and discussed the principal-axis coordinate system of the ${ }^{13} \mathrm{C}$ CSA tensor of the carboxylate carbon of L-alanine using their single crystal NMR results and the neutron diffraction data of Lehmann, Koetzle, and Hamilton [27]. A coordinate system defined by the $\mathrm{sp}^{2}$ symmetry of the carboxylate carbon was described with the z-axis perpendicular to the plane defined by the positions of the carboxylate 
carbon nucleus and the two oxygen nuclei, and the other two axes in this frame lie in the aforementioned plane with the $\mathrm{x}$-axis parallel to $\mathrm{C}(2)-\mathrm{C}(1)$ bond axis. Their experimentally determined CSA PAS differed slightly from the $\mathrm{sp}^{2}$-defined coordinate system. For example, the angles of the direction cosines between the z-axes and x-axes differed by $1.6^{\circ}$ and $9.4^{\circ}$, respectively.

Our focus is determining the orientation of the $\mathrm{N}-\mathrm{C}(1)$ internuclear vector in the CSA PAS. Two Euler angles of rotation $(\alpha, \beta)$ are required to bring the two respective $\mathrm{z}$ axes of the CSA tensor and the ${ }^{13} \mathrm{C}-{ }^{15} \mathrm{~N}$ dipolar tensor into alignment. Using the CSA PAS described by Naito et al. and the crystallographic data of Lehmann et al., the expected Euler angle pair is $\left(25^{\circ}, 80^{\circ}\right)$.

The ${ }^{13} \mathrm{C}-{ }^{15} \mathrm{~N}$ SEDOR difference experiment is as follows. The ${ }^{1} \mathrm{H}$ channel consists of a cross-polarization period followed by a strong decoupling field during the dipolar evolution period and during ${ }^{13} \mathrm{C}$ detection. The ${ }^{13} \mathrm{C}$ channel has a ${ }^{1} \mathrm{H}-{ }^{13} \mathrm{C}$ crosspolarization period, which is followed by a ${ }^{13} \mathrm{C}-{ }^{15} \mathrm{~N}$ dipolar evolution period of length $\tau$ containing a single $\square 180^{\circ}$ spin-echo pulse located at $\tau / 2$. In the absence of an ${ }^{15} \mathrm{~N}$ pulse there is no net ${ }^{13} \mathrm{C}-{ }^{15} \mathrm{~N}$ dipolar evolution during the dipolar evolution period, which yields the ${ }^{13} \mathrm{C}$ control spectrum. However, when a ${ }^{15} \mathrm{~N} 180^{\circ}$ pulse is applied at $\tau / 2$ a net dipolar dephasing of the ${ }^{13} \mathrm{C}$ spins occurs, which results in a less intense ${ }^{13} \mathrm{C}$ dephased spectrum having a unique dipolar modulated line shape.

Either the dephased spectrum or the difference spectrum (control minus dephased) provides sufficient spectral detail to determine the orientation of the N-C internuclear vector in the CSA PAS frame. For practical reasons, we focus the discussion on the difference spectrum. For example, if there are overlapping line shapes, then the difference spectrum will only contain contributions from the nuclei coupled to the heternuclear atom of interest.

Figure 1 shows simulations of difference spectra for different sets of the Euler angles using the NMR parameters provided in the Experimental section. The Euler angles are varied in $15^{\circ}$ increments from $0^{\circ}$ to $90^{\circ}$. Aside from the $\beta=0^{\circ}$ column (which represents alignment of the z-axes of the two tensors thereby making rotation by $\alpha$ irrelevant), a wide variety of line shapes with a strong dependence on the Euler angles is 
observed. This set of simulations suggests that the orientation of the internuclear vector can be easily extracted from analysis of difference line shapes.

Figure 2 shows the experimental control spectrum (left). The CSA-dominated control spectrum was used to obtain the CSA parameters. The experimental difference spectrum is presented on the right. Only the spectral region of the carboxylate ${ }^{13} \mathrm{C}$ line shape is shown in the two spectra. A simulation with the Euler angle pair $\left(26^{\circ}, 80^{\circ}\right)$ provided the best fit to the experimental spectrum and is shown as the dotted line superimposed over the experimental difference spectrum. This angle pair is similar to $\left(27^{\circ}, 80^{\circ}\right)$ found for L-alanine in the MAS NMR work of O'Connor and Schaefer [5]. Furthermore, our Euler angle pair is in excellent agreement with $\left(25^{\circ}, 80^{\circ}\right)$ predicted from the work of Naito et al.

Figure 3 shows a finer $(\alpha, \beta)$ grid in the region where the best fit to the experimental difference spectrum was found. The second row from the top is for $\alpha=26^{\circ}$, with the best fit to the experimental data being the $\beta=80^{\circ}$ simulation in the middle of this row. The remaining rows are for $\alpha$ values of $20^{\circ}, 25^{\circ}$, and $30^{\circ}$, and the columns are for $\beta$ values of $75^{\circ}, 80^{\circ}$, and $85^{\circ}$. Visual inspection of the $\alpha=26^{\circ}$ and $\alpha=25^{\circ}$ rows shows promise that it may be possible to determine $\alpha$ to within a degree. In any event, comparison of this set of simulations definitely suggests that $\alpha$ and $\beta$ values can be obtained to within $5^{\circ}$.

The experiments and simulations presented here were for a dipolar evolution period of 1.6 milliseconds. A survey of simulations shows that the overall features of the difference spectra for shorter evolution times are similar. For longer evolution times, the difference spectra do start to take on noticeably different shapes. Accordingly, one can imagine that if the dipolar coupling is not known or measured beforehand or if stronger evidence of the orientation is desired, then just a couple of difference spectra obtained with different dipolar evolution times would be useful. In fact, this suggests that a series of SEDOR-type measurements over many dipolar evolution times to simply determine the dipolar coupling before determining the relative orientation between the CSA and dipolar tensors is not necessary.

The results presented so far have shown that the orientation of the dipolar tensor in the CSA PAS can be determined accurately. However, a caveat needs to be 
mentioned. Magnetic susceptibility broadening can have a noticeable effect on the line shape of a spectrum obtained on a static solid sample [28-30]. In our case, we found that

the ${ }^{13} \mathrm{C}$ control spectrum of the alanine sample could not be satisfactorily simulated using the CSA parameters and only Lorentzian broadening. In order to get a good fit to the spectra a Gaussian broadening of approximately $300 \mathrm{~Hz}$ had to be included in the simulations, which we believe is necessary to compensate for magnetic susceptibility broadening of the line shape. While susceptibility broadening from the sample can be greatly reduced by magic angle spinning and the effects arising from probe geometry can be partly averaged away by MAS as well [31-33], it is necessary to keep the possible effects of magnetic susceptibility broadening in mind when working with static samples.

\section{Conclusion}

A single SEDOR difference spectrum can be used to determine the orientation of the vector connecting a nitrogen-carbon pair in the CSA PAS. The experiment should be useful when access to a MAS NMR probe is not available. The experiment may also be especially relevant for systems with small chemical shift anisotropies where it is difficult to generate enough spinning sidebands in MAS NMR for analysis. We envision use of the SEDOR experiment as a component in novel high-resolution solid-state NMR experiments that generate isotropic shifts in one dimension and dipole-modulated CSA powder patterns projected in the back dimension. For example, the experiment could be incorporated in the magic-angle hopping experiments and flipping of the spinning axis experiments of Bax, Szeverenyi, and Maciel $(34,35)$ and in the recoupling of chemical shift anisotropy experiments of Gullion (36), Chan and Tycko (37), Tycko, Dabbagh, and Mirau (38). There is potential of using the experiment in quadrupolar systems using the anisotropy of the quadrupole interaction instead of the CSA.

\section{$\underline{\text { Acknowledgements }}$}

The National Science Foundation supported this work through Grant CHE1152009. 


\section{$\underline{\text { Figure Captions }}$}

Figure 1. Simulated difference spectra over the full range of Euler angles $\alpha$ and $\beta$ in $15^{\circ}$ increments.

Figure 2. Experimental control spectrum of the L-alanine sample (left) and experimental difference spectrum superimposed over the best-fit simulation with Euler angles $\alpha=26^{\circ}$ and $\beta=80^{\circ}$ (right). The feature at the far right of the control spectrum is the onset of the contribution to the spectrum from the $-\mathrm{CH}$ carbon. The control spectrum is shown as $30 \%$ of its original height.

Figure 3. Fine grid difference simulations in the best-fit region of the experimental spectrum of the L-alanine sample. 


\section{$\underline{\text { References }}$}

1. G. D. Paepe, Dipolar recoupling in magic angle spinning solid-state nuclear magnetic resonance, Annu. Rev. Phys. Chem. 63 (2012) 661-684.

2. R. Tycko, Solid state nmr studies of amyloid fibril structure, Annu. Rev. Phys. Chem. 62 (2011) 279-299.

3. D. C. Apperley, R. K. Harris, and P. Hodgkinson, Solid State NMR: Basic Principles and Practice, Momentum Press, New York 2012.

4. J. M. Goetz and J. Schaefer, Orientational information in solids from REDOR sidebands, J. Magn. Reson. 129 (1997) 222-223.

5. R. D. O'Connor and J. Schaefer, Relative CSA-dipolar orientation from REDOR sidebands, J. Magn. Reson. 154 (2002) 46-52.

6. B. Heise, J. Leppert, and R. Ramachandran, Characterization of ${ }^{15} \mathrm{~N}-{ }^{13} \mathrm{C}$ chemical shift tensors and ${ }^{15} \mathrm{~N}-{ }^{1} \mathrm{H}$ dipolar-shift CPMAS NMR spectroscopy, Solid State Nucl. Magn. Reson. 16 (2000) 177-187.

7. J. Leppert, B. Heise, and R. Ramachandran, ${ }^{15} \mathrm{~N}$ chemical shift tensor magnitude and orientation in the molecular frame of uracil determined via MAS NMR, J. Magn. Reson. 145 (2000) 307-314.

8. F. G. Vogt, J. M. Gibson, S. M. Mattingly, and K. T. Mueller, Determination of molecular geometry in solid-state NMR: rotational-echo double resonance of three-spin systems, J. Phys. Chem. B 107 (2003) 1272-1283.

9. M. Zeyer, L. Montagne, and C. Jaeger, Measuring oxygen-phosphorus distances and the orientation of electric field gradient tensors using ${ }^{17} \mathrm{O}-\left\{{ }^{31} \mathrm{P}\right\}$ REDOR in phosphate glasses, Solid State Nucl. Magn. Reson. 23 (2003) 136-144. 
10. L. B. Andreas, A. K. Mehta, and M. A. Mehta, Determination of global structure from distance and orientation constraints in biological solids using solid-state NMR spectroscopy, J. Am. Chem. Soc. 129 (2007) 15233-25239.

11. C. M. Gowda, E. R. H. van Eck, A. M. van Buul, E. Schwartz, G. W. P. van Pruissen, J. J. L. M. Cornelissen, A. E. Rowan, R. J. M. Nolte, and A. P. M. Kentgens, Direct backbone structure determination of polyisocyanodipeptide using solid-state nuclear magnetic resonance, Macromolecules 45 (2012) 2209-2218.

12. T. Fujiwara, T. Shimomura, and H. Akutsu, Multidimensional solid-state nuclear magnetic resonance for correlating anisotropic interactions under magic-angle conditions, J. Magn. Reson. 124 (1997) 147-153.

13. Y. Ishii, T. Tereo, and M. Kainosho, Relayed anisotropy correlation NMR: determination of dihedral angles in solids, Chem. Phys. Lett. 256 (1996) 133-140.

14. D. M. Gregory, M. A. Mehta, J. C. Shiels, G. P. Drobny, Determination of local structure in solid nucleic acids using double quantum nuclear magnetic resonance spectroscopy, J. Chem. Phys. 107 (1997) 28-42.

15. R. Tycko, D. P. Weliky, and A. E. Berger, Investigation of molecular structure in solids by two-dimensional NMR exchange spectroscopy with magic angle spinning, J. Chem. Phys. 105 (1996) 7915-7930.

16. M. G. Munowitz, R. G. Griffin, G. Bodenhausen, and T. H. Huang, Twodimensional rotational spin-echo nuclear magnetic resonance in solids: Correlation of chemical shift and dipolar interaction, J. Am. Chem. Soc. 103 (1981) 2529-2533. 
17. H. Kaji and K. Schmidt-Rohr, Conformation and dynamics of atactic poly(acrylonitrile). 3. Characterization of local structure by two-dimensional ${ }^{2} \mathrm{H}^{13} \mathrm{C}$ solid-state NMR, Macromolecules 34 (2001) 7382-7391.

18. R. A. Haberkorn, R. E. Stark, H. van Willigen, and R. G. Griffin, Determination of bond distances and bond angles by solid-state nuclear magnetic resonance. ${ }^{13} \mathrm{C}$ and ${ }^{14}$ N NMR study of glycine, J. Am. Chem. Soc. 103 (1981) 2534-2539.

19. K. Schmidt-Rohr, Torsion angle determination in solid ${ }^{13} \mathrm{C}$-labeled amino acids and peptides by separated-local-field double-quantum NMR, J. Am. Chem. Soc. 118 (1996) 7601-7603.

20. M. E. Stoll, A. J. Vega, and R. W. Vaughan, Heteronuclear dipolar modulated chemical shift spectra for geometrical information in polycrystalline solids, J. Chem. Phys. 65 (1976) 4093-4098.

21. M. Linder, A. Hohener, and R. R. Ernst, Orientation of tensorial interactions determined from two-dimensional NMR powder spectra, J. Chem. Phys. 73 (1980) 49594970.

22. D. E. Kaplan and E. L. Hahn, Experiences de double irradiation en resonance magnetique par la method d'impulsions, J. Phys. Rad. 19 (1958) 821-825.

23. M. Emshwiller, E. L. Hahn, and D. Kaplan, Pulsed nuclear resonance spectroscopy, Phys. Rev. 118 (1960) 414-424.

24. D. V. Lang, J. B. Boyce, D. C. Lo, and C. P. Slichter, Measurement of electron spin density near Co atoms in Cu, Phys. Rev. Lett. 29 (1972) 776-779. 
25. M. Veshtort and R. G. Griffin, SPINEVOLUTION: A powerful tool for the simulation of solid and liquid state NMR experiments, J. Magn. Reson. 178 (2006) 248282.

26. A. Naito, S. Ganapathy, K. Akasaka, and C. A. McDowell, Chemical shielding tensor and ${ }^{13} \mathrm{C}-{ }^{14} \mathrm{~N}$ dipolar splitting in single crystals of L-alanine, J. Chem. Phys. 74 (1981) 3190-3197.

27. M. S. Lehmann, T. F. Koetzle, and W. C. Hamilton, Precision neutron diffraction structure determination of protein and nucleic acid components. I. The crystal and molecular structure of the amino acid L-alanine, J. Am. Chem. Soc. 94 (1972) 26572660.

28. L. E. Drain, The broadening of magnetic resonance lines due to field inhomogeneities in powdered samples, Proc. Phys. Soc. 80 (1962) 1380-1382.

29. M. E. Stoll and T. J. Majors, Reduction of Magnetic Susceptibility broadening in NMR by susceptibility matching, J. Magn. Reson. 46 (1982) 283-288.

30. A. N. Garroway, D. L. VanderHart, and W. L. Earl, ${ }^{13}$ C n.m.r. in organic solids: limits to spectral resolution and to determination of molecular motion, Phil. Trans. R. Lond. A 299 (1981) 609-628.

31. M. E. Stoll and T. J. Majors, Elimination of magnetic-susceptibility broadening in NMR using magic-angle sample spinning to measure chemical shifts in $\mathrm{NbH}_{\mathrm{x}}$, Phys. Rev. B 24 (1981) 2859-2862.

32. M. Alla and E. Lippmaa, Resolution limits in magic-angle rotation NMR spectra of polycrystalline solids, Chem. Phys. Lett. 87 (1982) 30-33. 
33. D. L. VanderHart, W. L. Earl, and A. N. Garroway, Resolution in ${ }^{13} \mathrm{C}$ NMR of organic solids using high-power proton decoupling and magic-angle sample spinning, J. Magn. Reson. 44 (1981) 361-401.

34. A. Bax, N. M. Szeverenyi, and G. E. Maciel, Correlation of isotropic shifts and chemical shift anisotropies by two-dimensional fourier-transform magic-angle hopping NMR spectroscopy, J. Magn. Reson. 52 (1983) 147-152.

35. A. Bax, N. M. Szeverenyi, and G. E. Maciel, Chemical shift anisotropy in powdered solids studied by 2D FT NMR with flipping of the spinning axis, J. Magn. Reson. 55 (1983) 494-497.

36. T. Gullion, Extended chemical-shift modulation, J. Magn. Reson. 85 (1989) 614619.

37. J. C. C. Chan and R. Tycko, Recoupling of chemical shift anisotropies in solidstate NMR under high-resolution magic-angle spinning and in uniformly ${ }^{13} \mathrm{C}$-labeled systems, J. Chem. Phys. 118 (2003) 8378-8389.

38. R. Tycko, G. Dabbagh, and P. A. Mirau, Determination of chemical-shiftanisotropy lineshapes in a two-dimensional magic-angle-spinning NMR experiment, J. Magn. Reson. 85 (1989) 265-274. 
Figure 1

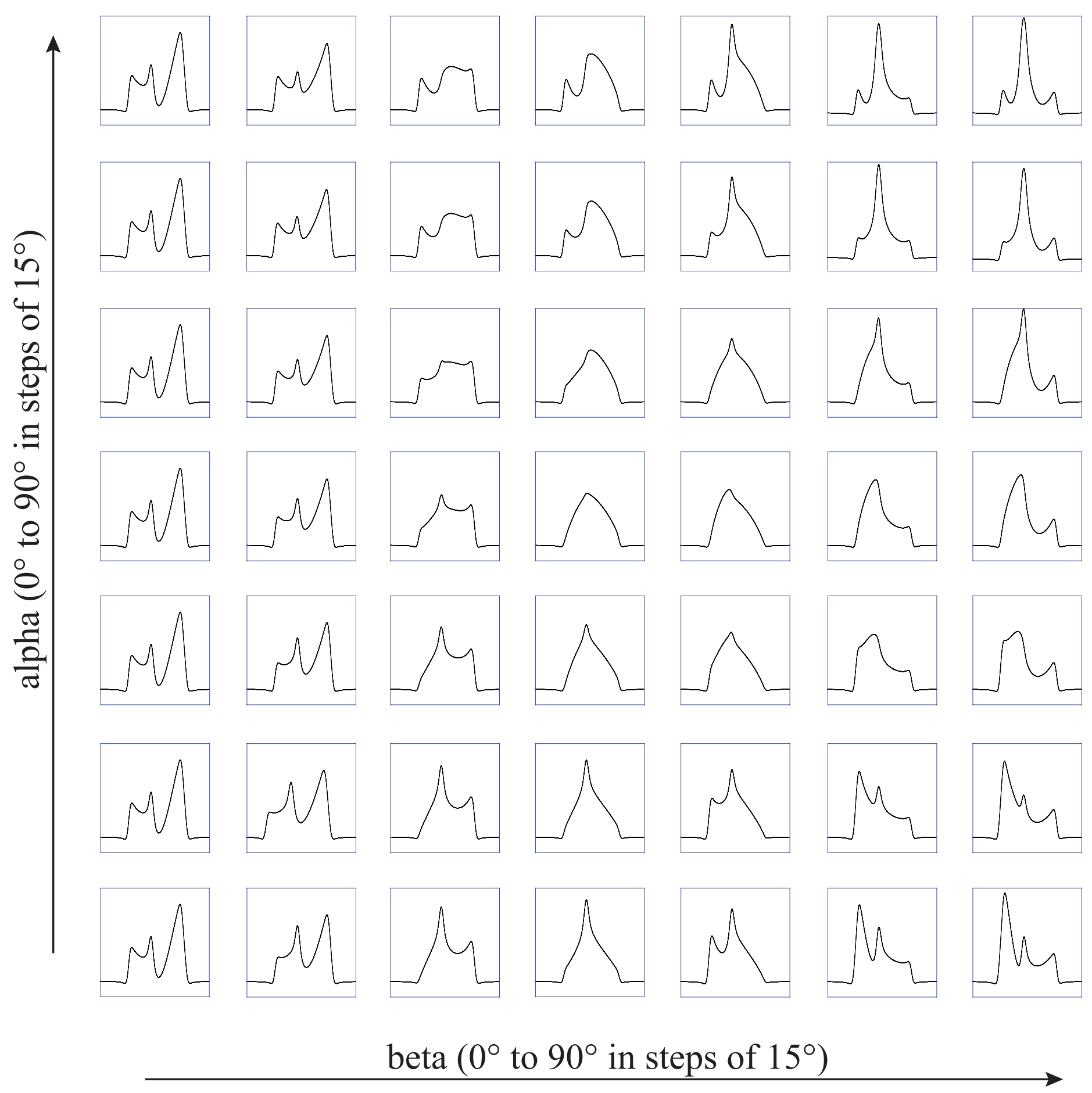




$$
\Lambda \Omega
$$


Figure 3

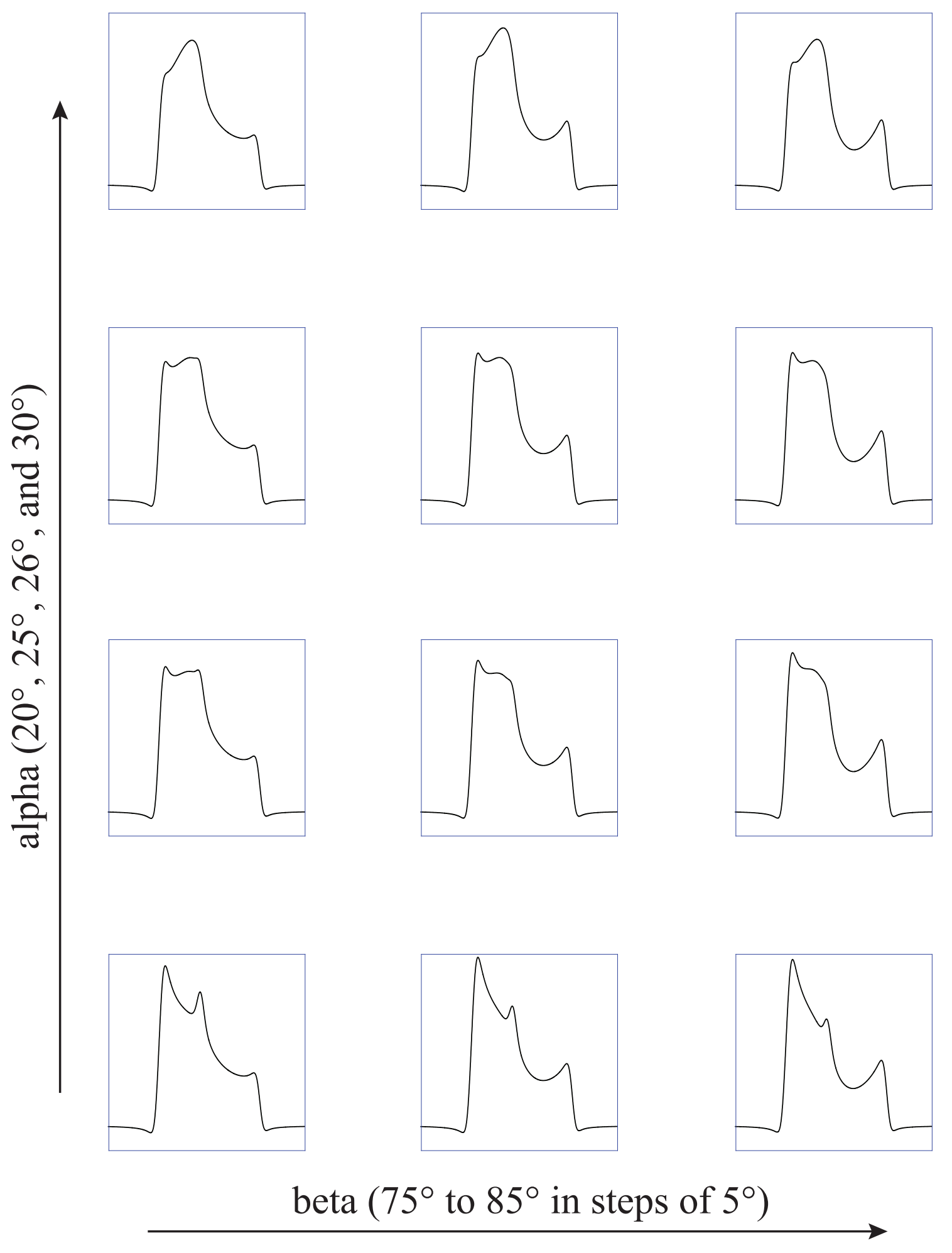




\section{Graphical Abstract}
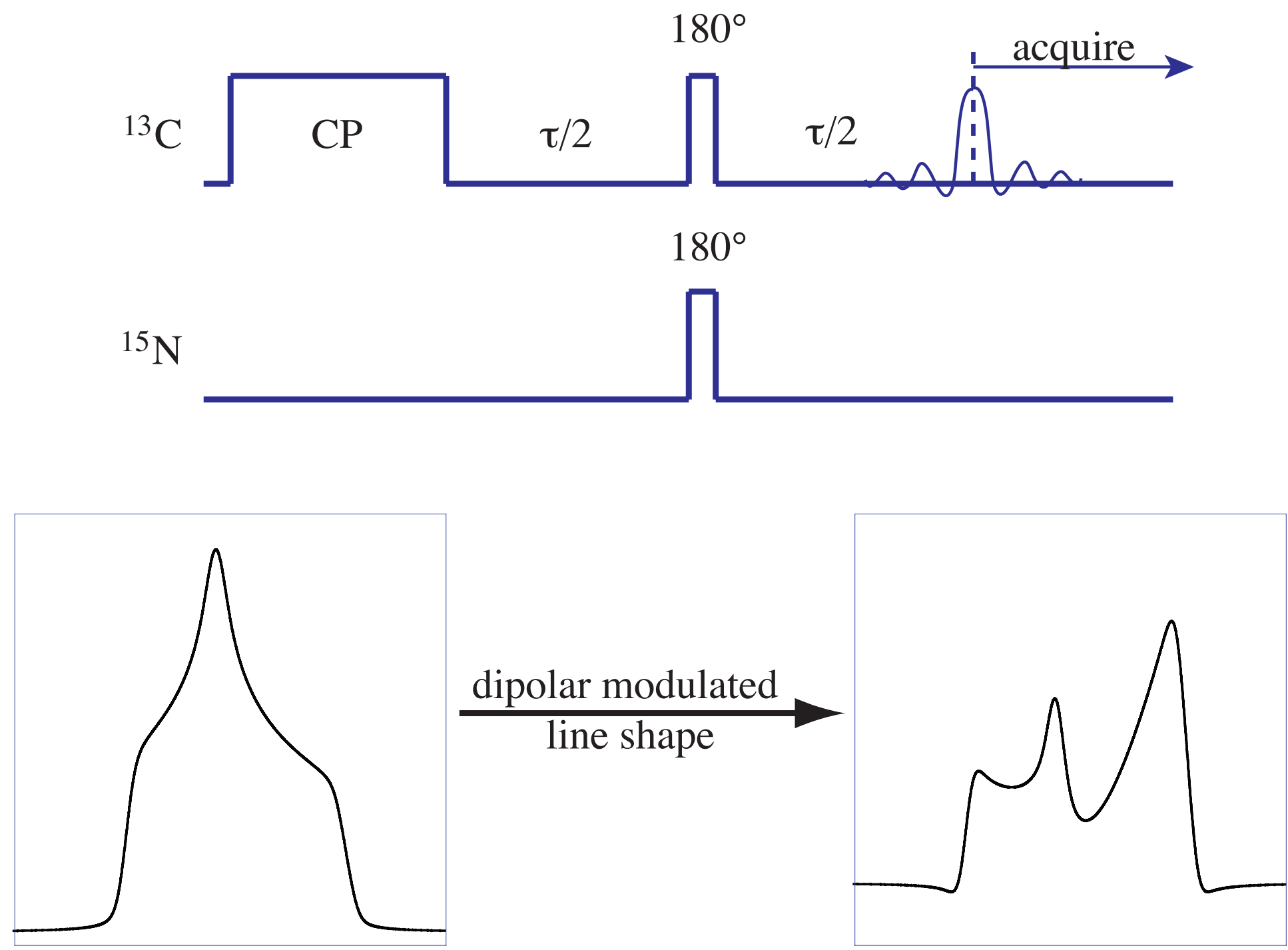